\title{
QUASI-SOCLE IDEALS IN BUCHSBAUM RINGS
}

\author{
SHIRO GOTO, JUN HORIUCHI, AND HIDETO SAKURAI
}

\begin{abstract}
Quasi-socle ideals, that is, ideals of the form $I=Q: \mathfrak{m}^{q}(q \geq 2)$, with $Q$ parameter ideals in a Buchsbaum local ring $(A, \mathfrak{m})$, are explored in connection to the question of when $I$ is integral over $Q$ and when the associated graded ring $\mathrm{G}(I)=\bigoplus_{n \geq 0} I^{n} / I^{n+1}$ of $I$ is Buchsbaum. The assertions obtained by Wang in the Cohen-Macaulay case hold true after necessary modifications of the conditions on parameter ideals $Q$ and integers $q$. Examples are explored.
\end{abstract}

\section{$\S 1$. Introduction}

Let $A$ be a Noetherian local ring with maximal ideal $\mathfrak{m}, d=\operatorname{dim} A>0$, and infinite residue class field $A / \mathfrak{m}$. Let

$$
\mathrm{G}(\mathfrak{m})=\bigoplus_{n \geq 0} \mathfrak{m}^{n} / \mathfrak{m}^{n+1}
$$

be the associated graded ring of $\mathfrak{m}$. For each $\mathfrak{m}$-primary ideal $I$ in $A$, we denote by $\mathrm{e}_{I}^{i}(A)(0 \leq i \leq d)$ the $i$ th Hilbert coefficient of $A$ with respect to $I$, whence the Hilbert polynomial of $I$ is given by the formula

$$
\ell_{A}\left(A / I^{n+1}\right)=\mathrm{e}_{I}^{0}(A)\left(\begin{array}{c}
n+d \\
d
\end{array}\right)-\mathrm{e}_{I}^{1}(A)\left(\begin{array}{c}
n+d-1 \\
d-1
\end{array}\right)+\cdots+(-1)^{d} \mathrm{e}_{I}^{d}(A)
$$

for all $n \gg 0$, where $\ell_{A}(*)$ denotes the length of the module.

With this notation, the purpose of this article is to prove the following.

THEOREM 1.1. Suppose that $A$ is a Buchsbaum local ring and suppose that depth $\mathrm{G}(\mathfrak{m}) \geq 2$. Let $q \geq 2$ be an integer, and let $Q=\left(a_{1}, a_{2}, \ldots, a_{d}\right)$ be a parameter ideal in $A$ such that $Q \subseteq \mathfrak{m}^{q+2}$. Assume that $a_{d}=a b$ for some $a \in \mathfrak{m}^{q}, b \in \mathfrak{m}$, and put $I=Q: \mathfrak{m}^{q}$. Then

$$
\mathfrak{m}^{q} I=\mathfrak{m}^{q} Q, \quad I \subseteq \mathfrak{m}^{q+2}, \quad \text { and } \quad I^{2}=Q I,
$$

and the following assertions hold true.

Received July 25, 2009. Revised February 11, 2010. Accepted February 15, 2010.

2000 Mathematics Subject Classification. Primary 13H10; Secondary 13A30, 13B22, $13 \mathrm{H} 15$.

(C) 2011 by The Editorial Board of the Nagoya Mathematical Journal 
(1) The first Hilbert coefficient $\mathrm{e}_{I}^{1}(A)$ of $A$ with respect to $I$ is given by $\mathrm{e}_{I}^{1}(A)=\mathrm{e}_{I}^{0}(A)+\mathrm{e}_{Q}^{1}(A)-\ell_{A}(A / I)$.

(2) The Hilbert function of $I$ is given by

$$
\begin{aligned}
\ell_{A}\left(A / I^{n+1}\right)= & \mathrm{e}_{I}^{0}(A)\left(\begin{array}{c}
n+d \\
d
\end{array}\right)-\mathrm{e}_{I}^{1}(A)\left(\begin{array}{c}
n+d-1 \\
d-1
\end{array}\right) \\
& +\sum_{i=2}^{d}(-1)^{i}\left[\mathrm{e}_{Q}^{i-1}(A)+\mathrm{e}_{Q}^{i}(A)\right]\left(\begin{array}{c}
n+d-i \\
d-i
\end{array}\right)
\end{aligned}
$$

for all $n \geq 0$.

(3) The associated graded ring $\mathrm{G}(I)=\bigoplus_{n \geq 0} I^{n} / I^{n+1}$ of $I$ is a Buchsbaum ring with

$$
\mathrm{H}_{M}^{i}(\mathrm{G}(I))=\left[\mathrm{H}_{M}^{i}(\mathrm{G}(I))\right]_{1-i} \cong \mathrm{H}_{\mathfrak{m}}^{i}(A)
$$

as $A$-modules for all $i<d$ and

$$
\max \left\{n \in \mathbb{Z} \mid\left[\mathrm{H}_{M}^{d}(\mathrm{G}(I))\right]_{n} \neq(0)\right\} \leq 1-d .
$$

Here $M=\mathfrak{m G}(I)+\mathrm{G}(I)_{+}$and $\left[\mathrm{H}_{M}^{i}(\mathrm{G}(I))\right]_{n}(i, n \in \mathbb{Z})$ denotes the homogeneous component with degree $n$ in the ith graded local cohomology module $\mathrm{H}_{M}^{i}(\mathrm{G}(I))$ of $\mathrm{G}(I)$ with respect to $M$.

Thus, the quasi-socle ideals $I=Q: \mathfrak{m}^{q}$ behave very well, inside Buchsbaum rings also, under the conditions stated in Theorem 1.1. Notice that, because $A$ is a Buchsbaum ring, the Hilbert coefficients $\mathrm{e}_{Q}^{i}(A)$ of the parameter ideal $Q$ are given by the formula

$$
(-1)^{i} \mathrm{e}_{Q}^{i}(A)= \begin{cases}\mathrm{e}_{Q}^{0}(A) & \text { if } i=0 \\
\ell_{A}\left(\mathrm{H}_{\mathfrak{m}}^{0}(A)\right) & \text { if } i=d, \\
\sum_{j=1}^{d-i}\left(\begin{array}{c}
d-i-1 \\
j-1
\end{array}\right) \ell_{A}\left(\mathrm{H}_{\mathfrak{m}}^{j}(A)\right) & \text { if } 1 \leq i \leq d-1\end{cases}
$$

and one has the equality $\ell_{A}\left(A / Q^{n+1}\right)=\sum_{i=0}^{d}(-1)^{i} \mathrm{e}_{Q}^{i}(A)\left(\begin{array}{c}n+d-i \\ d-1\end{array}\right)$ for all $n \geq 0$ (see [Sch, Korollar 3.2]), so that $\left\{\mathrm{e}_{Q}^{i}(A)\right\}_{1 \leq i \leq d}$ are independent of the choice of $Q$ and are invariants of $A$. The crucial point in Theorem 1.1 is the equality $I^{2}=Q I$; assertions (1), (2), and (3) readily follow from this fact via [GO, Section 2] and [GN, Section 5], since $\left(a_{1}, \ldots, \check{a}_{i}, \ldots, a_{d}\right): \mathfrak{m} \subseteq I$ for all $1 \leq i \leq d$. Here we should also note that the condition in Theorem 1.1 that 
$a_{d}=a b$ for some $a \in \mathfrak{m}^{q}$ and $b \in \mathfrak{m}$ is rather technical, but at this moment, we do not know whether this additional condition is superfluous.

We now briefly explain the background of Theorem 1.1. Our research dates back to works of A. Corso, C. Polini, C. Huneke, W. V. Vasconcelos, and the first author in which the socle ideals $Q: \mathfrak{m}$ for parameter ideals $Q$ in Cohen-Macaulay rings $A$ were explored with the following result.

TheOrem 1.2 (see [CHV], [CP1], [CP2], [CPV], [G2]). Let $Q$ be a parameter ideal in a Cohen-Macaulay ring $A$, and let $I=Q: \mathfrak{m}$. Then the following conditions are equivalent:

(1) $I^{2} \neq Q I$

(2) $Q$ is integrally closed in $A$;

(3) $A$ is a regular local ring, and the $A$-module $\mathfrak{m} / Q$ is cyclic.

Hence, if $A$ is a Cohen-Macaulay ring that is not regular, then $I^{2}=Q I$ for every parameter ideal $Q$ in $A$, so that $\mathrm{G}(I)$ and $\mathrm{F}(I)=\mathrm{G}(\mathrm{I}) / \mathfrak{m G}(\mathrm{I})$ are both Cohen-Macaulay rings, where $I=Q: \mathfrak{m}$. The Rees algebra $\mathcal{R}(I)=\bigoplus_{n \geq 0} I^{n}$ is also a Cohen-Macaulay ring, if $\operatorname{dim} A \geq 2$.

This result has led to two directions of research to better understand the quasi-socle ideals $I=Q: \mathfrak{m}^{q}$ in arbitrary local rings. One direction is to weaken the assumption on base rings $A$, which was performed by the first and the third authors (see [GSa1], [GSa2], [GSa3]). They explored the socle ideals $I=Q: \mathfrak{m}$ inside Buchsbaum local rings $A$ and showed that $I^{2}=Q I$ and that $\mathrm{G}(I)$ is a Buchsbaum ring if $\mathrm{e}_{\mathfrak{m}}^{0}(A) \geq 2$ and if $Q$ is contained in a sufficiently high power of the maximal ideal $\mathfrak{m}$. The other direction was independently performed by Wang [Wan] and also by the first author, Matsuoka, Takahashi, Kimura, Phuong, and Truong (see [GMT], [GKM], [GKMP], [GKPT] $)$. In [GMT] the quasi-socle ideals $Q: \mathfrak{m}^{2}$ in Gorenstein local rings $A$ with $\operatorname{dim} A>0$ and $\mathrm{e}_{\mathfrak{m}}^{0}(A) \geq 3$ are explored, and in [GKM], [GKMP], and [GKPT] the quasi-socle ideals $Q: \mathfrak{m}^{q}(q \geq 1)$ in Cohen-Macaulay local rings of dimension 1 are closely studied. However, at least in the case where $\operatorname{dim} A \geq 2$, Wang [Wan] made a great achievement in this area, settling affirmatively a conjecture of Polini and Ulrich [PU, page 663]. Let us note one of his results in the following form.

Theorem 1.3 ([Wan, Theorem 3.2]). Suppose that $A$ is a Cohen-Macaulay ring, and let $q \geq 1$ be an integer. Let $Q$ be a parameter ideal in $A$ such that $Q \subseteq \mathfrak{m}^{q+1}$, and put $I=Q: \mathfrak{m}^{q}$. Then

$$
\mathfrak{m}^{q} I=\mathfrak{m}^{q} Q, \quad I \subseteq \mathfrak{m}^{q+1}, \quad \text { and } \quad I^{2}=Q I,
$$


provided that depth $\mathrm{G}(\mathfrak{m}) \geq 2$.

Since Buchsbaum rings are very akin to Cohen-Macaulay rings, it seems quite natural to expect that similar results of the Cohen-Macaulay case, such as Theorem 1.3, should be true also in the Buchsbaum case after suitable modifications of the corresponding conditions, which we now report in Theorem 1.1 .

The proof of Theorem 1.1 is given in Section 2, which we divide into two parts. The first part shows that $\mathfrak{m}^{q} I=\mathfrak{m}^{q} Q$. The second part proves that $I^{2}=Q I$. Since $A$ is not necessarily a Cohen-Macaulay ring, the equality $I^{2}=Q I$ does not readily follow from the fact that $\mathfrak{m}^{q} I=\mathfrak{m}^{q} Q$. We carefully analyze this phenomenon in Section 2. A similar but more restricted result also holds true in the case where $G(\mathfrak{m})$ is a Buchsbaum ring with $\operatorname{depth} \mathrm{G}(\mathfrak{m})=1$, which we discuss in Section 3. In Section 4, we give examples of Buchsbaum rings $A$ with depth $\mathrm{G}(\mathfrak{m})=d-1$, which satisfy the conditions required in Theorems 1.1 and 3.1

In what follows, unless otherwise specified, let $(A, \mathfrak{m})$ denote a Noetherian local ring with $d=\operatorname{dim} A>0$. For simplicity, we assume throughout the article that the residue class field $A / \mathfrak{m}$ of $A$ is infinite. For an ideal $I$ in $A$, let

$$
\mathrm{G}(I)=\bigoplus_{n \geq 0} I^{n} / I^{n+1}
$$

be the associated graded ring of $I$. Let $\mathrm{H}_{\mathfrak{m}}^{i}(A)$ denote, for each $i \in \mathbb{Z}$, the $i$ th local cohomology module of $A$ with respect to $\mathfrak{m}$.

\section{§2. Proof of Theorem 1.1}

For each $f(\neq 0) \in A$, let $\mathrm{o}_{\mathfrak{m}}(f)=\max \left\{n \in \mathbb{Z} \mid f \in \mathfrak{m}^{n}\right\}$, and let $f^{*}=$ $f \bmod \mathfrak{m}^{n+1}$ denote the initial form of $f$ in $G(\mathfrak{m})$. The aim of this section is to prove Theorem 1.1. Let us begin with the following.

Lemma 2.1. Suppose that depth $\mathrm{G}(\mathfrak{m}) \geq 1$. Then $\mathfrak{m}^{\alpha}: \mathfrak{m}^{\beta}=\mathfrak{m}^{\alpha-\beta}$ for all $\alpha, \beta \in \mathbb{Z}$ with $\beta \geq 0$.

Proof. We have to show only that $\mathfrak{m}^{\alpha}: \mathfrak{m}^{\beta} \subseteq \mathfrak{m}^{\alpha-\beta}$. Let us choose $a \in$ $\mathfrak{m} \backslash \mathfrak{m}^{2}$ so that $a^{*}$ is $\mathrm{G}(\mathfrak{m})$-regular. Let $x \in \mathfrak{m}^{\alpha}: \mathfrak{m}^{\beta}$. Assume that $x \notin \mathfrak{m}^{\alpha-\beta}$. Then, since $\mathrm{o}_{\mathfrak{m}}(x) \leq \alpha-\beta-1$ and $a^{\beta} x \in \mathfrak{m}^{\alpha}$, we have $a^{* \beta} x^{*}=0$ in $\mathrm{G}(\mathfrak{m})$, which is impossible.

After suitable modifications of conditions on parameter ideals $Q$ and integers $q$, Wang's [Wan] technique still works in the case where $A$ is a gener- 
alized Cohen-Macaulay ring. We summarize this fact in the following, for which we note a detailed proof in order to clarify where and why we need such modifications.

Proposition 2.2. Suppose that depth $\mathrm{G}(\mathfrak{m}) \geq 2$. Assume that $A$ is a generalized Cohen-Macaulay ring, and choose an integer $\ell>0$ so that $\mathfrak{m}^{\ell}$ is standard (see [T, Section 3]). Let $q \geq 2$ be an integer, and let $Q=\left(a_{1}, a_{2}, \ldots\right.$, $\left.a_{d}\right)$ be a parameter ideal in $A$ such that $Q \subseteq \mathfrak{m}^{q+\ell+1}$. We put $I=Q: \mathfrak{m}^{q}$. Then $\mathfrak{m}^{q} I=\mathfrak{m}^{q} Q$ and $I \subseteq \mathfrak{m}^{q+\ell+1}$, whence $I^{2} \subseteq Q$.

Proof. Once we have $\mathfrak{m}^{q} I=\mathfrak{m}^{q} Q$, by Lemma 2.1 we get that $I \subseteq \mathfrak{m}^{q} Q$ : $\mathfrak{m}^{q} \subseteq \mathfrak{m}^{2 q+\ell+1}: \mathfrak{m}^{q}=\mathfrak{m}^{q+\ell+1}$. We now show that $\mathfrak{m}^{q} I \subseteq \mathfrak{m}^{q} Q$

Let $F$ denote the set of all the products $\prod_{i=1}^{q} f_{i}$, where $f_{i} \in \mathfrak{m} \backslash \mathfrak{m}^{2}$ for all $1 \leq i \leq q$ and $f_{i}^{*}, f_{j}^{*}$ form a regular sequence in $\mathrm{G}(\mathfrak{m})$ for all integers $1 \leq i<j \leq q$. Then $\mathfrak{m}^{q}=(F)$. Let $\alpha \in I$, and let $f=\prod_{i=1}^{q} f_{i} \in F$, where the family $\left\{f_{i}\right\}_{1 \leq i \leq q}$ of elements in $\mathfrak{m} \backslash \mathfrak{m}^{2}$ is assumed to satisfy the conditions stated above. Let us write

$$
\alpha f=\sum_{i=1}^{d} a_{i} x_{i}
$$

with $x_{i} \in A$. It suffices to show that $x_{i} \in \mathfrak{m}^{q}$ for all $1 \leq i \leq d$.

We put $g_{j}=\prod_{1 \leq k \leq q, k \neq j} f_{k}$ for each $1 \leq j \leq q$, and we choose $g \in \mathfrak{m} \backslash \mathfrak{m}^{2}$ so that $g^{*}, f_{j}^{*}$ is a regular sequence in $\mathrm{G}(\mathfrak{m})$ for all $1 \leq j \leq q$. Let

$$
\alpha\left(g_{j} g\right)=\sum_{i=1}^{d} a_{i} x_{i j}
$$

with $x_{i j} \in A$. Then, since $f=f_{j} g_{j}$, we have

$$
\sum_{i=1}^{d} a_{i}\left(f_{j} x_{i j}\right)=\sum_{i=1}^{d} a_{i}\left(g x_{i}\right),
$$

whence, for all $1 \leq i \leq d$ and $1 \leq j \leq q$, we get

$$
g x_{i}-f_{j} x_{i j} \in\left(a_{1}, \ldots, \check{a}_{i}, \ldots, a_{d}\right): a_{i} .
$$

Therefore, since $\left(a_{1}, \ldots, \check{a_{i}}, \ldots, a_{d}\right): a_{i}=\left(a_{1}, \ldots, \check{a_{i}}, \ldots, a_{d}\right): \mathfrak{m}^{\ell}$ by [T, Proposition 3.1] (recall that $\mathfrak{m}^{\ell}$ is standard) and $Q: \mathfrak{m}^{\ell} \subseteq \mathfrak{m}^{q+\ell+1}: \mathfrak{m}^{\ell}=\mathfrak{m}^{q+1}$ by Lemma 2.1, we get

$$
g x_{i}-f_{j} x_{i j} \in \mathfrak{m}^{q+1}
$$


Consequently, since $g^{*}, f_{j}^{*}$ form a regular sequence in $\mathrm{G}(\mathfrak{m})$, we have

$$
g x_{i}-f_{j} x_{i j} \in\left(g, f_{j}\right) \cap \mathfrak{m}^{q+1}=\left(g, f_{j}\right) \cdot \mathfrak{m}^{q}
$$

$($ see $[\mathrm{VV}])$, so that $g x_{i}-f_{j} x_{i j}=g x_{i}^{\prime}-f_{j} x_{i j}^{\prime}$ with $x_{i}^{\prime}, x_{i j}^{\prime} \in \mathfrak{m}^{q}$. Hence,

$$
x_{i}-x_{i}^{\prime} \in\left(f_{j}\right): g=\left(f_{j}\right),
$$

and so $x_{i} \in \mathfrak{m}^{q}+\left(f_{j}\right)$ for all $1 \leq i \leq d$ and $1 \leq j \leq q$. Thus,

$$
x_{i} \in \bigcap_{j=1}^{q}\left[\mathfrak{m}^{q}+\left(f_{j}\right)\right] .
$$

CLAIM 1. $\bigcap_{j=1}^{k}\left[\mathfrak{m}^{q}+\left(f_{j}\right)\right] \subseteq \mathfrak{m}^{q}+\left(\prod_{j=1}^{k} f_{j}\right)$ for all $1 \leq k \leq q$.

Proof. We may assume that $k>1$ and that our assertion holds true for $k-1$. Hence,

$$
\bigcap_{j=1}^{k}\left[\mathfrak{m}^{q}+\left(f_{j}\right)\right] \subseteq\left[\mathfrak{m}^{q}+\left(\prod_{j=1}^{k-1} f_{j}\right)\right] \cap\left[\mathfrak{m}^{q}+\left(f_{k}\right)\right]=\mathfrak{m}^{q}+\left[\left(\prod_{j=1}^{k-1} f_{j}\right) \cap\left[\mathfrak{m}^{q}+\left(f_{k}\right)\right]\right] .
$$

Let $y \in A$, and assume that $x=\left(\prod_{j=1}^{k-1} f_{j}\right) \cdot y \in \mathfrak{m}^{q}+\left(f_{k}\right)$. Let $\bar{A}=A /\left(f_{k}\right)$, and let $\overline{\mathfrak{m}}=\mathfrak{m} /\left(f_{k}\right)$. Let $\bar{*}$ denote the image in $\bar{A}$. Then $\bar{y} \in \overline{\mathfrak{m}}^{q-(k-1)}$, because $\overline{\prod_{j=1}^{k-1} f_{j}} \cdot \bar{y} \in \overline{\mathfrak{m}}^{q}$ and $f_{j}^{*}, f_{k}^{*}$ form a regular sequence in $\mathrm{G}(\mathfrak{m})$ for all $1 \leq j \leq$ $k-1$. Hence, $y \in \mathfrak{m}^{q-k+1}+\left(f_{k}\right)$, so that $x=\left(\prod_{j=1}^{k-1} f_{j}\right) \cdot y \in \mathfrak{m}^{q}+\left(\prod_{j=1}^{k} f_{j}\right)$. Thus, $\left(\prod_{j=1}^{k-1} f_{j}\right) \cap\left[\mathfrak{m}^{q}+\left(f_{k}\right)\right] \subseteq \mathfrak{m}^{q}+\left(\prod_{j=1}^{k} f_{j}\right)$, whence

$$
\bigcap_{j=1}^{k}\left[\mathfrak{m}^{q}+\left(f_{j}\right)\right] \subseteq \mathfrak{m}^{q}+\left(\prod_{j=1}^{k} f_{j}\right)
$$

Thanks to Claim 1 , we get $x_{i} \in \mathfrak{m}^{q}+\left(\prod_{j=1}^{q} f_{j}\right)=\mathfrak{m}^{q}$ for all $1 \leq i \leq d$, whence $\alpha f \in \mathfrak{m}^{q} Q$, which proves Proposition 2.2.

We need the following to show the equality $I^{2}=Q I$.

Lemma 2.3 ([GSa3, Lemma 2.3]). Let $W, L$, and $M$ be ideals in a commutative ring $R$, and let $a, b \in R$. Assume that $a \in M, a W=(0), L: a=L: a^{2}$, and $L: a b=L: b$. Then

$$
(L+(a b)+W): M=[(L+W): M]+[(L+(a b)): M] .
$$


If $L: a=L: M$, we furthermore have

$$
(L+(a b)+W): M=(L+(a b)): M .
$$

Proof. The former assertion is proved exactly in the same way as [GSa3, Lemma 2.3], where we have no utility of the assumption that $b \in M$. To see the latter equality, let $x \in(L+W): M$. Then, since $a^{2} x \in L$, we get

$$
x \in L: a^{2}=L: a=L: M \subseteq(L+(a b)): M .
$$

Thus, $(L+W): M \subseteq(L+(a b)): M$, whence

$$
(L+(a b)+W): M=[(L+W): M]+(L+(a b)): M=(L+(a b)): M,
$$

as required.

The heart of our proof of Theorem 1.1 is the following.

Proposition 2.4. Suppose that $A$ is a generalized Cohen-Macaulay ring, and choose an integer $\ell>0$ so that $\mathfrak{m}^{\ell}$ is standard. Let $q \geq 1$ be an integer, and let $Q=\left(a_{1}, a_{2}, \ldots, a_{d}\right)$ be a parameter ideal in $A$. Let $I=Q: \mathfrak{m}^{q}$, and assume that the following three conditions are satisfied:

(1) $\mathfrak{m}^{q} I=\mathfrak{m}^{q} Q, I^{2} \subseteq Q$, and $a_{i} \in \mathfrak{m}^{\ell}$ for all $1 \leq i \leq d-1$;

(2) there exist elements $a \in \mathfrak{m}^{q}$ and $b \in \mathfrak{m}$ such that $a_{d}=a b$ and both systems $\left\{a_{1}, a_{2}, \ldots, a_{d-1}, a\right\}$ and $\left\{a_{1}, a_{2}, \ldots, a_{d-1}, b\right\}$ of parameters in $A$ are standard;

(3) either $d=1$ or $q \geq \ell$.

We then have $I^{2}=Q I$.

Proof. We notice that the system $\left\{a_{1}, a_{2}, \ldots, a_{d}\right\}$ of parameters is standard, because $\left\{a_{1}, a_{2}, \ldots, a_{d-1}, a\right\}$ is also standard (see [T, Corollary 3.3]). We put $W=\mathrm{H}_{\mathfrak{m}}^{0}(A), L=\left(a_{1}, a_{2}, \ldots, a_{d-1}\right)$ and $M=\mathfrak{m}^{q}$. Then $a \in M, a W=$ $(0)$, and

$$
L: a=L: a^{2}=L: a b=L: b=\bigcup_{n \geq 0}\left[L: \mathfrak{m}^{n}\right],
$$

since all the systems $\left\{a_{1}, a_{2}, \ldots, a_{d-1}, a\right\},\left\{a_{1}, a_{2}, \ldots, a_{d-1}, b\right\}$, and $\left\{a_{1}\right.$, $\left.a_{2}, \ldots, a_{d-1}, a b\right\}$ of parameters are standard. On the other hand, we have $\bigcup_{n \geq 0}\left[L: \mathfrak{m}^{n}\right]=L: \mathfrak{m}^{\ell}$ by $\left[\mathrm{T}\right.$, Proposition 3.1] because $L \subseteq \mathfrak{m}^{\ell}$ and $\mathfrak{m}^{\ell}$ is standard. Hence, $L: a=L: M$, if $q \geq \ell$. Consequently, since $W=W: M$, by Lemma 2.3 we get

$$
(Q+W): \mathfrak{m}^{q}=W+\left[Q: \mathfrak{m}^{q}\right]=W+I,
$$


if $d=1$, and

$$
(Q+W): \mathfrak{m}^{q}=Q: \mathfrak{m}^{q}=I,
$$

if $q \geq \ell$.

Suppose now that $d=1$, and let $\bar{A}=A / W, \overline{\mathfrak{m}}=\mathfrak{m} / W, \bar{I}=I \bar{A}$, and $\bar{Q}=Q \bar{A}$. Then $\bar{I}=\bar{Q}: \overline{\mathfrak{m}}^{q}$ and $\overline{\mathfrak{m}}^{q} \cdot \bar{I}=\overline{\mathfrak{m}}^{q} \cdot \bar{Q}$. Let $x \in \bar{I}^{2}$. Then, since $\bar{I}^{2} \subseteq \bar{Q}$, we have $x=a_{1} y$ with $y \in \bar{A}$. Let $\alpha \in \overline{\mathfrak{m}}^{q}$. Then, since $a_{1}(\alpha y)=\alpha x \in$ $\overline{\mathfrak{m}}^{q} \cdot \bar{I}^{2}=\overline{\mathfrak{m}}^{q} \cdot \bar{Q}^{2}$, we get $a_{1}(\alpha y)=a_{1}^{2} z$ for some $z \in \bar{A}$. Therefore, $\alpha y \in \bar{Q}$ (notice that $a_{1}$ is $\bar{A}$-regular), so that $x=a_{1} y \in \bar{Q} \cdot \bar{I}$, because $y \in \bar{Q}: \overline{\mathfrak{m}}^{q}=\bar{I}$. Thus, $\bar{I}^{2}=\bar{Q} \cdot \bar{I}$, so that $I^{2} \subseteq Q I+W$. Since $W \cap Q=(0)$ and $I^{2} \subseteq Q$, we get $I^{2} \subseteq(Q I+W) \cap Q=Q I$, as required.

Suppose now that $d \geq 2$ and that our assertion holds true for $d-1$. Let $B=A /\left(a_{1}\right)$. Then all the conditions (1), (2), and (3) are satisfied for the parameter ideal $Q /\left(a_{1}\right)$ in $B$, and we get $I^{2} \subseteq Q I+\left(a_{1}\right)$. Let $x \in I^{2}$, and write $x=y+a_{1} z$ with $y \in Q I$ and $z \in A$. Let $\alpha \in \mathfrak{m}^{q}$. We then have

$$
\alpha x=\alpha y+a_{1}(\alpha z) \in Q^{2},
$$

because $x \in I^{2}$ and $\mathfrak{m}^{q} I=\mathfrak{m}^{q} Q$. Consequently, $a_{1}(\alpha z) \in Q^{2}$ (notice that $\left.\alpha y \in Q^{2}\right)$, so that $a_{1}(\alpha z) \in\left(a_{1}\right) \cap Q^{2}=a_{1} Q$, because $a_{1}, a_{2}, \ldots, a_{d}$ form a $d$-sequence in $A$ (see [T, Proposition 3.1]). Hence, $\alpha z-v \in(0): a_{1} \subseteq W$ for some $v \in Q$, which guarantees that $z \in(Q+W): \mathfrak{m}^{q}=I$, since $q \geq \ell$. Thus, $x=y+a_{1} z \in Q I$, so that $I^{2}=Q I$ as claimed.

Summarizing Propositions 2.2 and 2.4, we have the following. Taking $\ell=1$ in the case where $A$ is a Buchsbaum ring, Theorem 1.1 now follows from Theorem 2.5.

TheOREM 2.5. Suppose that $A$ is a generalized Cohen-Macaulay ring and suppose that depth $\mathrm{G}(\mathfrak{m}) \geq 2$. Choose an integer $\ell \geq 1$ so that $\mathfrak{m}^{\ell}$ is standard. Let $Q=\left(a_{1}, a_{2}, \ldots, a_{d}\right)$ be a system of parameters in $A$, and put $I=Q: \mathfrak{m}^{q}$, where $q$ is an integer such that $q \geq \max \{\ell, 2\}$. Assume that the following two conditions are satisfied:

(i) $Q \subseteq \mathfrak{m}^{q+\ell+1}$;

(ii) there exist elements $a \in \mathfrak{m}^{q}$ and $b \in \mathfrak{m}$ such that $a_{d}=a b$ and the system $a_{1}, \ldots, a_{d-1}, b$ of parameters in $A$ is standard.

Then $I^{2}=Q I$, and the following assertions hold true:

(1) $\mathrm{e}_{I}^{1}(A)=\mathrm{e}_{I}^{0}(A)+\mathrm{e}_{Q}^{1}(A)-\ell_{A}(A / I)$; 
(2) the Hilbert function of I is given by

$$
\begin{aligned}
\ell_{A}\left(A / I^{n+1}\right) & =\mathrm{e}_{I}^{0}(A)\left(\begin{array}{c}
n+d \\
d
\end{array}\right)-\mathrm{e}_{I}^{1}(A)\left(\begin{array}{c}
n+d-1 \\
d-1
\end{array}\right) \\
& +\sum_{i=2}^{d}(-1)^{i}\left[\mathrm{e}_{Q}^{i-1}(A)+\mathrm{e}_{Q}^{i}(A)\right]\left(\begin{array}{c}
n+d-i \\
d-i
\end{array}\right)
\end{aligned}
$$

for all $n \geq 0$;

(3) the graded ring $\mathrm{G}(I)=\bigoplus_{n>0} I^{n} / I^{n+1}$ is Buchsbaum, if $A$ is also;

(4) $\mathrm{H}_{M}^{i}(\mathrm{G}(I))=\left[\mathrm{H}_{M}^{i}(\mathrm{G}(I))\right]_{1-i} \cong \mathrm{H}_{\mathfrak{m}}^{i}(A)$ as $A$-modules for all $i<d$ and

$$
\max \left\{n \in \mathbb{Z} \mid\left[\mathrm{H}_{M}^{d}(\mathrm{G}(I))\right]_{n} \neq(0)\right\} \leq 1-d,
$$

where $M=\mathfrak{m G}(I)+\mathrm{G}(I)_{+}$.

Proof. The equality $I^{2}=Q I$ follows directly from Propositions 2.2 and 2.4. See [GO, Section 2] (resp., [GN, Section 5]) for assertions (1) and (2) (resp., (3) and (4)).

\section{$\S 3$. The case where $\operatorname{depth} \mathrm{G}(\mathfrak{m})=1$}

In this section, we study the question of what happens in the case where $\operatorname{depth} \mathrm{G}(\mathfrak{m})=1$. Our goal is the following.

TheOREM 3.1. Let $A$ be a Buchsbaum ring with $d=\operatorname{dim} A \geq 2$, and suppose that $\mathrm{G}(\mathfrak{m})$ is a Buchsbaum ring with $\operatorname{depth} \mathrm{G}(\mathfrak{m})=1$. Let

$$
n=\min \left\{n \in \mathbb{Z} \mid\left[\mathrm{H}_{M}^{1}(\mathrm{G}(\mathfrak{m}))\right]_{n} \neq(0)\right\},
$$

where $M=\mathrm{G}(\mathfrak{m})_{+}$. Then $n \geq 0$, and for every integer $1 \leq q \leq n+1$ and for every parameter ideal $Q=\left(a_{1}, a_{2}, \ldots, a_{d}\right)$ of $A$ such that $Q \subseteq \mathfrak{m}^{q+2}$ we have

(1) $\mathfrak{m}^{q} I=\mathfrak{m}^{q} Q$, and

(2) $I \subseteq \mathfrak{m}^{q+2}$,

where $I=Q: \mathfrak{m}^{q}$. Consequently, $I^{2}=Q I$, so that assertions (1), (2), and (3) in Theorem 1.1 hold true also in the present setting, provided that $a_{d}=$ $a b$ for some $a \in \mathfrak{m}^{q}$ and $b \in \mathfrak{m}$.

The proof of Theorem 3.1 is essentially the same as that of Theorem 1.1. However, we note a detailed proof to show where we use the assumption that $1 \leq q \leq n+1$. 
Proof. Choose $f \in \mathfrak{m} \backslash \mathfrak{m}^{2}$ so that $f^{*}$ is $\mathrm{G}(\mathfrak{m})$-regular. Then, since $\mathrm{G}(\mathfrak{m})$ is a Buchsbaum ring, we get

$$
\mathrm{H}_{M}^{0}(\mathrm{G}(\overline{\mathfrak{m}})) \cong\left[\mathrm{H}_{M}^{1}(\mathrm{G}(\mathfrak{m}))\right](-1)
$$

as graded $\mathrm{G}(\mathfrak{m})$-modules, where $\overline{\mathfrak{m}}=\mathfrak{m} /(f)$. Hence,

$$
n+1 \geq \min \left\{n \in \mathbb{Z} \mid\left[\mathrm{H}_{M}^{0}(\mathrm{G}(\overline{\mathfrak{m}}))\right]_{n} \neq(0)\right\} \geq 1,
$$

so that $n \geq 0$.

To show assertion (1), we may assume that $q \geq 2$ (see [GSa1], [GSa2], [GSa3] for the case where $q=1$ ). Let $F$ denote the set of all the products $\prod_{i=1}^{q} f_{i}$, where $f_{i} \in \mathfrak{m} \backslash \mathfrak{m}^{2}$ for all $1 \leq i \leq q$ and $f_{i}^{*}, f_{j}^{*}$ form, for all integers $1 \leq i<j \leq q$, a part of a homogeneous system of parameters in $\mathrm{G}(\mathfrak{m})$. Then $\mathfrak{m}^{q}=(F)$. Let $\alpha \in I$, and let $f=\prod_{i=1}^{q} f_{i} \in F$, where the family $\left\{f_{i}\right\}_{1 \leq i \leq q}$ of elements in $\mathfrak{m} \backslash \mathfrak{m}^{2}$ is assumed to satisfy the conditions stated above. Let us write

$$
\alpha f=\sum_{i=1}^{d} a_{i} x_{i},
$$

with $x_{i} \in A$. We will now show that $x_{i} \in \mathfrak{m}^{q}$ for all $1 \leq i \leq d$.

We put $g_{j}=\prod_{1 \leq k \leq q, k \neq j} f_{k}$ for each $1 \leq j \leq q$, and we choose $g \in \mathfrak{m} \backslash \mathfrak{m}^{2}$ so that $g^{*}, f_{j}^{*}$ is a part of a homogeneous system of parameters in $\mathrm{G}(\mathfrak{m})$ for all $1 \leq j \leq q$. Let

$$
\alpha\left(g_{j} g\right)=\sum_{i=1}^{d} a_{i} x_{i j},
$$

with $x_{i j} \in A$. Then, since $f=f_{j} g_{j}$, we have

$$
\sum_{i=1}^{d} a_{i}\left(f_{j} x_{i j}\right)=\sum_{i=1}^{d} a_{i}\left(g x_{i}\right),
$$

whence, for all $1 \leq i \leq d$ and $1 \leq j \leq q$, we get

$$
g x_{i}-f_{j} x_{i j} \in\left(a_{1}, \ldots, \check{a}_{i}, \ldots, a_{d}\right): a_{i} .
$$

Therefore, since $\left(a_{1}, \ldots, \check{a_{i}}, \ldots, a_{d}\right): a_{i}=\left(a_{1}, \ldots, \check{a_{i}}, \ldots, a_{d}\right): \mathfrak{m}$ and $Q: \mathfrak{m} \subseteq$ $\mathfrak{m}^{q+1}$ by Lemma 2.1 , we have

$$
g x_{i}-f_{j} x_{i j} \in \mathfrak{m}^{q+1} .
$$


Let $\bar{A}=A /\left(f_{j}\right)$, and let $\bar{*}$ denote the image in $\bar{A}$. We then have

$$
\bar{g} \cdot \overline{x_{i}} \in \overline{\mathfrak{m}}^{q+1}
$$

where $\overline{\mathfrak{m}}=\mathfrak{m} /\left(f_{j}\right)$. Hence, $\overline{x_{i}} \in \overline{\mathfrak{m}}^{q}$. In fact, assume that $\overline{x_{i}} \notin \overline{\mathfrak{m}}^{q}$, and let $\ell=\mathrm{o}_{\overline{\mathfrak{m}}}\left(\overline{x_{i}}\right)$. Then $\ell \leq q-1$, while

$$
0 \neq{\overline{x_{i}}}^{*} \in \mathrm{H}_{M}^{0}(\mathrm{G}(\overline{\mathfrak{m}})) \cong\left[\mathrm{H}_{M}^{1}(\mathrm{G}(\mathfrak{m}))\right](-1)
$$

Hence, $\left[\mathrm{H}_{M}^{1}(\mathrm{G}(\mathfrak{m}))\right]_{\ell-1} \neq(0)$, and so $n \leq \ell-1 \leq q-2$. This is impossible, since $q \leq n+1$ by our assumption.

Thus, $x_{i} \in \mathfrak{m}^{q}+\left(f_{j}\right)$ for all $1 \leq i \leq d$ and $1 \leq j \leq q$, so that the proof of Claim 1 shows that $x_{i} \in \mathfrak{m}^{q}$ for all $1 \leq i \leq d$. In fact, with the same notation as in the proof of Claim 1, the crucial point is to check that $\bar{y} \in \overline{\mathfrak{m}}^{q-(k-1)}$. Suppose that $\bar{y} \notin \overline{\mathfrak{m}}^{q-(k-1)}$. Then, since $\overline{\prod_{j=1}^{k-1} f_{j}} \cdot \bar{y} \in \overline{\mathfrak{m}}^{q}$ and $\left(\overline{\prod_{j=1}^{k-1} f_{j}}\right)^{*}$ is a part of a homogeneous system of parameters in the Buchsbaum ring $\mathrm{G}(\overline{\mathfrak{m}})$, we get $\bar{y}^{*} \in \mathrm{H}_{M}^{0}(\mathrm{G}(\overline{\mathfrak{m}}))$, so that $n+1 \leq \mathrm{o}_{\overline{\mathfrak{m}}}(\bar{y}) \leq q-k \leq q-2$, which is impossible, since $q \leq n+1$. Hence, $\mathfrak{m}^{q} I=\mathfrak{m}^{q} Q$, so that $I \subseteq \mathfrak{m}^{q+2}$ by Lemma 2.1. The other assertions follow similarly as in the proof of Theorem 1.1.

\section{§4. Example}

Let $d>0$ and $n \geq 0$ be integers. We look at the graded ring

$$
R=k\left[X_{1}, X_{2}, \ldots, X_{d}, Y_{1}, Y_{2}, \ldots, Y_{d}\right] /\left[\left(Y_{i} \mid 1 \leq i \leq d\right)^{n+2}+\left(\sum_{i=1}^{d} X_{i} Y_{i}^{n+1}\right)\right]
$$

where $U=k\left[X_{1}, X_{2}, \ldots, X_{d}, Y_{1}, Y_{2}, \ldots, Y_{d}\right]$ denotes the polynomial ring with $2 d$ indeterminates over a field $k$. Let $M=R_{+}, A=R_{M}$, and $\mathfrak{m}=M R_{M}$.

EXAMPLE 4.1. The following assertions hold true:

(1) $\operatorname{dim} R=d$ and $\operatorname{depth} R=d-1$;

(2) $\mathrm{H}_{M}^{d-1}(R) \cong[R / M](-(n+2-d))$ as graded $R$-modules;

(3) $R$ is a Buchsbaum ring;

(4) $\mathrm{e}_{\mathfrak{m}}^{0}(A)=\left(\begin{array}{c}d+n+1 \\ d\end{array}\right)-1$.

Since $R \cong \mathrm{G}(\mathfrak{m})$, Example 4.1 provides Buchsbaum rings $A$ which satisfy the conditions required in Theorem 1.1 (take $d \geq 3$ ) and Theorem 3.1 (take $d=2)$. 
Proof. Let $\mathfrak{p}=\left(Y_{i} \mid 1 \leq i \leq d\right)$, and let $f=\sum_{i=1}^{d} X_{i} Y_{i}^{n+1}$. Let $\mathfrak{a}=\mathfrak{p}^{n+2}+$ $(f)$, and let $V=U / \mathfrak{p}$. We look at the two short exact sequences

$$
\begin{aligned}
& \text { (1) } 0 \rightarrow \mathfrak{p}^{n+1} / \mathfrak{a} \rightarrow R \rightarrow U / \mathfrak{p}^{n+1} \rightarrow 0, \\
& \text { (2) } 0 \rightarrow \mathfrak{a} / \mathfrak{p}^{n+2} \stackrel{\varphi}{\rightarrow} \mathfrak{p}^{n+1} / \mathfrak{p}^{n+2} \rightarrow \mathfrak{p}^{n+1} / \mathfrak{a} \rightarrow 0
\end{aligned}
$$

of graded $R$-modules. Then we see that $\mathfrak{p}^{n+1} / \mathfrak{p}^{n+2}$ is a free $V$-module with $\left\{Y^{\alpha} \bmod \mathfrak{p}^{n+2}\right\}_{\alpha \in \Lambda}$ a basis, where

$$
\Lambda=\left\{\left(\alpha_{1}, \alpha_{2}, \ldots, \alpha_{d}\right) \mid 0 \leq \alpha_{i} \in \mathbb{Z} \text { for } 1 \leq \forall i \leq d, \sum_{i=1}^{d} \alpha_{i}=n+1\right\}
$$

and $Y^{\alpha}=\prod_{i=1}^{d} Y_{i}^{\alpha_{i}}$ for each $\alpha=\left(\alpha_{1}, \alpha_{2}, \ldots, \alpha_{d}\right) \in \Lambda$. Notice that

$$
\mathfrak{a} / \mathfrak{p}^{n+2} \cong(f) /\left[(f) \cap \mathfrak{p}^{n+2}\right] \cong(f) / f \mathfrak{p} \cong V(-(n+2))
$$

Then, identifying $\mathfrak{a} / \mathfrak{p}^{n+2}=V(-(n+2))$ and $\mathfrak{p}^{n+1} / \mathfrak{p}^{n+2}=V^{q}(-(n+1))$ with $q=\left(\begin{array}{c}n+d \\ d-1\end{array}\right)$, we see that the inclusion map $\varphi: \mathfrak{a} / \mathfrak{p}^{n+2} \rightarrow \mathfrak{p}^{n+1} / \mathfrak{p}^{n+2}$ in exact sequence $(2)$ is represented by the matrix of the form

$$
\left(\begin{array}{c}
X_{1} \bmod \mathfrak{p} \\
X_{2} \bmod \mathfrak{p} \\
\vdots \\
X_{d} \bmod \mathfrak{p} \\
0 \\
\vdots \\
0
\end{array}\right),
$$

so that we have

$\mathfrak{p}^{n+1} / \mathfrak{a} \cong V^{q-d}(-(n+1)) \oplus\left[\operatorname{Syz}_{V}^{d-1}\left(V /\left(X_{1}, X_{2}, \ldots, X_{d}\right) V\right)\right](-(n+2-d))$, where $\operatorname{Syz}_{V}^{d-1}\left(V /\left(X_{1}, X_{2}, \ldots, X_{d}\right) V\right)$ denotes the $(d-1)$ th syzygy module of the residue class field $V /\left(X_{1}, X_{2}, \ldots, X_{d}\right) V$ of $V$. Hence, $\operatorname{depth}_{R} L=d-1$, where $L=\mathfrak{p}^{n+1} / \mathfrak{a}$, and

$$
\mathrm{H}_{M}^{d-1}(L) \cong(R / M)(-(n+2-d))
$$

as graded $R$-modules. Consequently, thanks to exact sequence (1), we get $\operatorname{depth} R=d-1$ and

$$
\mathrm{H}_{M}^{d-1}(R) \cong \mathrm{H}_{M}^{d-1}(L) \cong(R / M)(-(n+2-d))
$$


because $U / \mathfrak{p}^{n+1}$ is a Cohen-Macaulay ring of dimension $d$. Thus, $R$ is a Buchsbaum ring by [SV, Corollary 1.1]. We have

$$
\mathrm{e}_{\mathfrak{m}}^{0}(A)=\ell_{U_{\mathfrak{p}}}\left(R_{\mathfrak{p}}\right)=\left(\begin{array}{c}
n+d+1 \\
d
\end{array}\right)-1
$$

by the associative formula of multiplicity, since $\operatorname{Min}_{U} R=\{\mathfrak{p}\}$.

REMARK 4.2. Taking $n=0$ in Example 4.1, we have

$$
\mathrm{e}_{\mathfrak{m}}^{0}(A)=d=1+\sum_{i=1}^{d-1}\left(\begin{array}{c}
d-1 \\
i-1
\end{array}\right) h^{i}(A),
$$

where $h^{i}(A)=\ell_{A}\left(\mathrm{H}_{\mathfrak{m}}^{i}(A)\right)$. Hence, our Buchsbaum local ring $A$ has minimal multiplicity in the sense of [G1, Section 4].

\section{REFERENCES}

[CHV] A. Corso, C. Huneke, and W. V. Vasconcelos, On the integral closure of ideals, Manuscripta Math. 95 (1998), 331-347.

[CP1] A. Corso and C. Polini, Links of prime ideals and their Rees algebras, J. Algebra 178 (1995), 224-238.

[CP2] , Reduction number of links of irreducible varieties, J. Pure Appl. Algebra 121 (1997), 29-43.

[CPV] A. Corso, C. Polini, and W. V. Vasconcelos, Links of prime ideals, Math. Proc. Cambridge Philos. Soc. 115 (1994), 431-436.

[G1] S. Goto, On the associated graded rings of parameter ideals in Buchsbaum rings, J. Algebra 85 (1983), 490-534.

[G2] - Integral closedness of complete intersection ideals, J. Algebra 108 (1987), 151-160.

[GKM] S. Goto, S. Kimura, and N. Matsuoka, Quasi-socle ideals in Gorenstein numerical semigroup rings, J. Algebra 320 (2008), 276-293.

[GKMP] S. Goto, S. Kimura, N. Matsuoka, and T. T. Phuong, Quasi-socle ideals in local rings with Gorenstein tangent cones, J. Commut. Algebra 1 (2009), 603-620.

[GKPT] S. Goto, S. Kimura, T. T. Phuong, and H. L. Truong, Quasi-socle ideals and Goto numbers of parameters, J. Pure Appl. Algebra 214 (2010), 501-511.

[GMT] S. Goto, N. Matsuoka, and R. Takahashi, Quasi-socle ideals in a Gorenstein local ring, J. Pure Appl. Algebra 212 (2008), 969-980.

[GN] S. Goto and K. Nishida, Hilbert coefficients and Buchsbaumness of associated graded rings, J. Pure Appl. Algebra 181 (2003), 61-74.

[GO] S. Goto and K. Ozeki, The structure of Sally modules - towards a theory of nonCohen-Macaulay cases, preprint, 2009.

[GSa1] S. Goto and H. Sakurai, The equality $I^{2}=Q I$ in Buchsbaum rings, Rend. Sem. Mat. Univ. Padova 110 (2003), 25-56.

[GSa2] - The reduction exponent of socle ideals associated to parameter ideals in a Buchsbaum local ring of multiplicity two, J. Math. Soc. Japan 56 (2004), $1157-1168$. 
[GSa3] When does the equality $I^{2}=Q I$ hold true in Buchsbaum rings?, Lect. Notes Pure Appl. Math. 244 (2006), 115-139.

[PU] C. Polini and B. Ulrich, Linkage and reduction numbers, Math. Ann. 310 (1998), 631-651.

[Sch] P. Schenzel, Multiplizitäten in verallgemeinerten Cohen-Macaulay-Moduln, Math. Nachr. 88 (1979), 295-306.

[SV] J. Stückrad and W. Vogel, Toward a theory of Buchsbaum singularities, Amer. J. Math. 100 (1978), 727-746.

[T] N. V. Trung, Toward a theory of generalized Cohen-Macaulay modules, Nagoya Math. J. 102 (1986), 1-49.

[VV] P. Valabrega and G. Valla, Form rings and regular sequences, Nagoya Math. J. 72 (1978), 93-101.

[Wan] H.-J. Wang, Links of symbolic powers of prime ideals, Math. Z. 256 (2007), 749-756.

Shiro Goto

Department of Mathematics

School of Science and Technology

Meiji University

Kawasaki 214-8571

Japan

goto@math.meiji.ac.jp

Jun Horiuchi

Department of Mathematics

School of Science and Technology

Meiji University

Kawasaki 214-8571

Japan

jhoriuchi@math.meiji.ac.jp

Hideto Sakurai

Department of Mathematics

School of Science and Technology

Meiji University

Kawasaki 214-8571

Japan

hsakurai@math.meiji.ac.jp 